\title{
Prevalencia de Corynebacterium spp. y factores asociados en mujeres del Valle de Aburrá
}

\author{
Prevalence of corynebacterium spp. and \\ associated factors in women of Valle de Aburrá
}

\author{
Adriana María Botero, Xiomara Úsuga, Claudia María Cuervo, \\ Ana Claudia Ossa - Medellín (Colombia)
}

\section{Resumen}

Introducción: Corynebacterium spp. está presente en flujo vaginal de mujeres asintomáticas, pero se ha encontrado asociado a procesos patológicos, generando confusión al momento de clasificarlo como microbiota o microorganismos patógenos. Nuestro objetivo fue determinar la prevalencia de Corynebacterium spp. y explorar su asociación con características clínicas y hábitos sexuales.

Material y métodos: estudio descriptivo transversal en 511 mujeres del Valle de Aburrá durante 2012 y 2013. Los datos demográficos, clínicos y de comportamiento sexual se obtuvieron mediante encuestas; la información sobre el perfil microbiológico genital se obtuvo de muestra de flujo vaginal. El análisis descriptivo se hizo con frecuencias y medidas de resumen; para el análisis bivariado se usó chi cuadrado, prueba exacta de Fisher, U de Mann Whitney y se usó regresión logística para el análisis multivariado; los análisis se realizaron en el programa estadístico IBM SPSS Statistics versión 22.

Resultados: la prevalencia de Corynebacterium spp fue 59\%. Referente al comportamiento sexual durante el último mes previo a la toma de muestra, encontramos que las participantes tuvieron sexo con una persona en promedio (rango de 0 - 3 personas distintas); respecto a las prácticas durante el coito en el mismo mes, se observó que $58 \%$ de las mujeres tuvo sexo sin preservativo, a $61 \%$ le practicaron sexo oral y a $10 \%$ sexo anal. Se encontró asociación de Corynebacterium spp con reacción leucocitaria.

Conclusiones: la prevalencia de Corynebacterium spp fue 59\% y se encontró asociado a reacción leucocitaria; no se asoció a comportamientos sexuales específicos ni sintomatología ginecológica. (Acta Med Colomb 2015; 40: 234-240).

Palabras clave: Corynebacterium, flujo vaginal, mujeres, microbiota, Colombia.

\footnotetext{
Abstract

Introduction: Corynebacterium spp. is present in vaginal fluid of asymptomatic women, but it has been found associated with disease processes, creating confusion when classified as normal or pathogenic flora. Our objective was to determine the prevalence of Corynebacterium spp. and explore its association with clinical characteristics and sexual habits.

Materials and Methods: descriptive cross-sectional study in 511 women of Valle de Aburrá in 2012 and 2013. Demographic, clinical and sexual behavioral data were obtained through surveys; information about genital microbiological profile was obtained from sample of vaginal discharge. Descriptive analysis was done with frequencies and summary measures; for bivariate analysis chi square, Fisher exact test and Mann Whitney U test were used, and logistic regression was used for multivariate analysis; analysis were performed in the statistical program SPSS Statistics version 22.

Results: The prevalence of Corynebacterium spp was 59\%. Regarding sexual behavior during the last pre-sampling month, we found that participants had sex with a person on average (range from 0-3 different people); regarding practices during intercourse in the same month, it was observed
}

Dra. Adriana María Botero: Bacterióloga, Estudiante Posgrado, Institución Universitaria Colegio Mayor de Antioquia; Dra. Xiomara Úsuga: MSc., Bacterióloga y Laboratorista Clínico, Grupo Infettare, Facultad de Medicina, Universidad Cooperativa de Colombia sede Medellín; Dra. Claudia María Cuervo: MSc., Bacterióloga y Laboratorista Clínico, Grupo de Investigación Biociencias, Facultad Ciencias de la Salud, Institución Universitaria Colegio Mayor de Antioquia; Dra. Ana Claudia Ossa: Especialista, Microbióloga y Bioanalista, Grupo Infettare, Facultad de Medicina, Universidad Cooperativa de Colombia sede Medellín; Grupo de Investigación Biociencias, Facultad Ciencias de la Salud, Institución Universitaria Colegio Mayor de Antioquia. Medellín (Colombia). Correspondencia. Dra. Ana Claudia OssaGiraldo. Medellín (Colombia).

E-mail: ana.ossag@campusucc.edu.co, ana ossa@colmayor.edu.co

Recibido: 11/XI/2014 Aceptado: 21/IX/2015 
that $58 \%$ of women had sex without a condom, to $61 \%$ oral sex was practiced and to $10 \%$ anal sex.

Association of Corynebacterium spp with leukocyte reaction was found.

Conclusions: The prevalence of Corynebacterium spp was 59\% and was found associated with leukocyte reaction; it was not associated to specific sexual behaviors or gynecological symptoms.

(Acta Med Colomb 2015; 40: 234-240).

Keywords: Corynebacterium, vaginal discharge, women, microbiota, Colombia.

\section{Introducción}

El tracto genital femenino tiene una flora normal que la defiende de microorganismos patógenos, entre los cuales se encuentran en mayor porcentaje Lactobacillus spp (1-4), que tienen la capacidad de producir ácido láctico, peróxido de hidrógeno y bacteriocinas que protegen la mucosa genital (5); en menor porcentaje se encuentran otros microorganismos que en ocasiones pueden ser patógenos como Streptococcus grupo B, Escherichia coli, Mycoplasma spp., Mobiluncus spp., Peptoestreptococcus spp., Gardnerella spp. y hongos $(6,7)$.

La disminución de las especies de Lactobacillus spp., favorecen el incremento de otros microorganismos tales como Gardnerella vaginalis, Prevotella spp., Bacteroides spp, Mobiluncus spp., y Candida spp., causando enfermedad a nivel genital (8-14). Especies del género Corynebacterim también se encuentran frecuentemente en tracto genitourinario de mujeres, en algunas ocasiones reportadas como flora normal, y en otras, asociadas a infección o procesos patológicos.

Las especies de Corynebacterium diferentes a $C$. diphtheriae, conocidas como difteroides o bacterias corineformes, son reconocidas por ser bacilos gram positivos, pleomórficos, inmóviles, no esporulados, productoras de catalasa y formadoras de gránulos metacromáticos, algunas aerobias exclusivas y en su mayoría anaerobias facultativas (15 - 18). Su identificación morfológica se realiza mediante la tinción de Gram, en la cual se observan bacilos gram positivos (algunos teñidos irregularmente), pleomórficos dado que algunos son rectos y otros ligeramente curvados, con extremos cónicos o curvos, y con orientación angular de letra " $v$ " o letras chinas (debido a la división celular) o en empalizada $(16,17,19$ - 21).

Las bacterias corineformes o difteroides fueron consideradas durante mucho tiempo microorganismos con poca significancia clínica dado que no causaban infecciones graves al humano, sino que hacían parte de la flora saprófita de la piel humana y las mucosas (16); no obstante, cada día se descubren aspectos de patogenicidad de estos microorganismos, capaces de sobrevivir por largo tiempo en el ambiente hospitalario $(15,17)$, ocasionando problemas de salud en pacientes inmunosuprimidos (22), en pacientes con dispositivos protésicos (23) y en aquellos con estancias prolongadas en hospitales, ocasionando enfermedades como linfadenitis granulomatosa, neumonitis (C. striatum), faringitis, infecciones de la piel, endocarditis en pacientes con dispositivos intravasculares (C. amycolatum) y prostatitis $(C$. glucuronolyticum $)$, entre otros, $(15,22$ - 25), razones que hacen considerarlas como patógenos oportunistas.

Igualmente varias especies de bacterias corineformes se han encontrado asociadas a infecciones en el tracto genitourinario femenino y masculino como es el caso de $C$. riegelii y $C$. urealyticum; e implicadas en complicaciones del embarazo como C. aurimucosum (22), mientras que otras especies difteroides han sido aisladas de este mismo sitio anatómico y halladas en fluidos corporales, sin certeza sobre su patogenicidad o simple presencia como $C$. freneyi (esperma, y tracto genital femenino), C. glucuronolyticum (semen y tracto genital masculino), C. lipophiloflavum (secreción vaginal), C. singulare (semen), C. tuberculostericum (uretra, orina, tracto urogenital), y C. macginleyi y C. pseudodiphtheriticum (orina) (22).

Desde el año 1997, la Sociedad Americana de Microbiología (ASM, del inglés American Society of Microbiology) publicó los criterios para asociar la presencia de bacterias corineformes con enfermedad. Uno de estos corresponde a encontrar en una muestra clínica teñida con Gram la presencia concomitante de bacilos gram positivos con morfología y orientación compatible con difteroides y una fuerte reacción leucocitaria, entre otros criterios relacionados con los hallazgos en cultivos y pruebas bioquímicas (25).

En la actualidad es difícil encontrar criterios unificados acerca de la interpretación clínica de la presencia de Corynebacterium spp. en flujo vaginal si se tiene en cuenta que los pocos estudios a nivel mundial sobre este microorganismo presentan discrepancias, toda vez que unos reportan esta bacteria como flora acompañante en vagina y otros la asocian a procesos patológicos.

Por lo anterior y teniendo en cuenta la llamativa frecuencia del reporte de estos microorganismos en flujo vaginal en nuestro medio, por ejemplo, una prevalencia de $32 \%$ observada en un estudio que realizamos en pacientes que asistían a una institución prestadora de servicios de salud en Medellín durante 2006 - 2012 (26), se decidió determinar la prevalencia de Corynebacterium spp. en mujeres de población general y explorar asociaciones con los factores demográficos, clínicos y de comportamiento sexual.

\section{Material y métodos}

Estudio descriptivo transversal en 511 mujeres mayores de edad del Valle de Aburrá, durante 2012 y 2013, muestreadas a conveniencia. Los datos demográficos, clínicos y de comportamiento sexual se obtuvieron por fuente primaria 
a través de encuestas aplicadas por personal previamente capacitado; para controlar los sesgos de información se realizó una prueba piloto y validez de apariencia y contenido a las encuestas por expertos. En la sistematización de los datos se realizaron auditorías a la información registrada. Se incluyeron en este estudio mujeres mayores de 18 años, gestantes y no gestantes residentes del Valle de Aburrá,Antioquia. No se aceptaron aquellas mujeres que no firmaron el consentimiento informado, quienes presentaron sangrado menstrual al momento de la toma de la muestra, mujeres menores de edad y aquellas que nunca hubieran tenido relaciones sexuales.

\section{Procedimientos bioquímicos y microbiológicos}

\section{Toma de muestras}

Para realizar la toma de muestras de flujo vaginal se colocó un espéculo nuevo y estéril (Dispositivos Médicos Procitol) en el canal cervical de cada participante, se localizó el cuello uterino y se observaron las características del flujo vaginal tomando registro de la cantidad, aspecto y color del mismo. Seguidamente se recolectó muestra de endocérvix introduciendo un citocepillo (Dispositivos Médicos Procitol) en el canal endocervical, rotándolo 180 grados; esta muestra fue extendida en forma circular en un portaobjetos nuevo. Con un aplicador estéril de algodón se tomó una muestra de exocérvix y se extendió en el portaobjetos en forma vertical al lado de la muestra de endocérvix previamente dispuesta; una vez secas ambas muestras, el portaobjetos fue almacenado para su posterior tinción con la coloración de Gram. Luego de realizar los extendidos, el aplicador de algodón fue depositado en un tubo con solución salina estéril para la realización del examen directo. Con un segundo aplicador estéril se recolectó muestra de fondo de saco para el análisis del test de aminas y finalmente se tomó un fragmento de cinta indicadora de $\mathrm{pH}$ específica para rango ácido (PH Indicator $\mathrm{pH} 4-7$, marca Whatman $\left.{ }^{\circledR}\right)$, la cual se frotó directamente contra la pared vaginal y para realizar la respectiva lectura del $\mathrm{pH}$ vaginal. Todos los implementos de toma de muestras fueron nuevos, estériles y se usaron una única vez por participante.

\section{Test de aminas}

Inmediatamente luego de la toma de muestra de fondo de vagina, se agregaron dos gotas de hidróxido de potasio $(\mathrm{KOH})$ al $10 \%$ al aplicador estéril que contenía dicha muestra y se procedió a identificar el olor generado, clasificando como positivo para test de aminas aquella muestra en la que se desprendiera un olor "a pescado", característico de la presencia de aminas producidas por bacterias anaerobias.

\section{Medición de pH vaginal}

El pH vaginal se cuantificó con un fragmento de cinta indicadora de $\mathrm{pH}$ específica para rango ácido ( $\mathrm{PH}$ Indicator pH 4-7, marca Whatman ${ }^{\circledR}$ ) para garantizar la identificación de pequeñas fluctuaciones de $\mathrm{pH}$ vaginal que no son posibles de detectar con la cinta medidora de $\mathrm{pH}$ de uso común (rango entre 3 y 9 aproximadamente). Este fragmento de aproximadamente tres centímetros de longitud fue frotado directamente contra la pared vaginal y como lo indica el fabricante, el valor de $\mathrm{pH}$ se asignó de acuerdo con el color que tomó la cinta luego del contacto con la muestra.

\section{Examen directo}

Luego de la recolección, una gota de la muestra de exocérvix en solución salina estéril fue depositada en un portaobjetos nuevo y cubierta con una laminilla cubreobjetos y fue analizada en microscopio óptico de luz con el objetivo de 40X para la identificación de la presencia células guía, Trichomonas spp. y pseudomicelios.

\section{Coloración de Gram}

Las muestras de flujo vaginal fueron coloreadas con la tinción de Gram (IHR Diagnostica Ltda) de acuerdo con las instrucciones del fabricante; la lectura se realizó en microscopio óptico de luz con el objetivo de 100X. Se revisaron diez campos y se hizo un promedio de los microorganismos y células observadas reportándose de la siguiente manera: escaso (de 1 - 5), moderado (5 - 10) y abundante (más de 10). Para garantizar la confiabilidad del diagnóstico, la lectura fue realizada por dos observadores distintos y posteriormente se evaluó su concordancia.

\section{Diagnóstico de vaginosis bacteriana}

La vaginosis bacteriana (VB) fue diagnosticada de acuerdo con los criterios de Amsel. Se clasificó como positiva para VB aquella participante que cumplía por lo menos tres de los cuatro criterios: (a) flujo vaginal blanco o gris, fino y homogéneo, (b) presencia de células guía al examen directo, (c) $\mathrm{pH}$ del flujo vaginal $>4.5 \mathrm{y}$ (d) test de aminas positivo. Del total de las participantes, se analizaron 281 para VB en quienes se realizó la medición de $\mathrm{pH}$.

\section{Análisis estadísticos}

La información se sistematizó en una base de datos en el programa Microsoft Excel 2013 y fue analizada en el software estadístico IBM SPSS Statistics versión 22. El análisis descriptivo se hizo con frecuencias y medidas de resumen; para el análisis bivariado se usó chi cuadrado, prueba exacta de Fisher y U de Mann Whitney; se usó regresión logística para el análisis multivariado. El valor de $\mathrm{P}<0.05$ se consideró significativo.

\section{Aspectos éticos}

Todos los procedimientos de esta investigación se realizaron siguiendo los lineamientos de la Resolución 8430 de 1993 y la Declaración de Helsinki, y para su ejecución se contó con la aprobación del Subcomité de Bioética en Investigación de la Universidad Cooperativa de Colombia sede Medellín. 


\section{Resultados}

El promedio de edad de la población estudiada fue 26 años (desviación estándar 10, mediana 22, rango intercuartil 19-29), la mayoría pertenecía a los estratos socioeconómicos 2,3 y $4(30,44$ y $12 \%$ respectivamente) y $57 \%$ cursaba o había culminado estudios de pregrado, seguido de $23 \%$ que tenía estudios técnicos. Respecto a los métodos anticonceptivos, los más usados eran: orales (29\%), condón (24\%) e inyectables (16\%); sólo $9 \%$ de la población dijo usar duchas vaginales.

A las mujeres se les indagó al momento de la toma de muestras si presentaban síntomas ginecológicos y el personal encargado de tomar la muestra de flujo verificó el aspecto y color de éste. En la Tabla 1 se muestran los principales hallazgos de estas características.

La prevalencia de bacterias corineformes en esta población fue $59 \%$. El microorganismo más frecuente fue el género Lactobacillus, seguido de difteroides, y cocobacilos gram variables compatibles con Gardnerella spp. El 92\% de las mujeres presentaron algún grado de reacción leucocitaria y $21-16 \%$ presentaron células guía y test de aminas positivo respectivamente (Tabla 2 ).

Referente al comportamiento sexual durante el último mes previo a la toma de muestra, encontramos que las participantes tuvieron sexo con una persona en promedio (rango de 0 - 3 personas distintas), y al indagar sobre las prácticas durante el coito en el mismo mes, se observó que $58 \%$ de las mujeres tuvieron sexo sin preservativo, a $61 \%$ le practicaron sexo oral y $10 \%$ sexo anal.

Se exploró la posible asociación entre la presencia de Corynebacterium spp. y las variables clínicas y sexuales. De acuerdo con este análisis bivariado, se halló asociación con color del flujo, pH, test de aminas, células guía, lactobacilos, reacción leucocitaria, hongos, cocobacilos gram variables, Mobiluncus spp., y VB (Tabla 3). No se halló asociación con síntomas ginecológicos ni hábitos sexuales. Al hacer el modelo de regresión logística de las anteriores variables, sólo la reacción leucocitaria continuó presentando asociación estadísticamente significativa con las bacterias corineformes $(\mathrm{p}=0.017)$.

\section{Discusión}

En los flujos vaginales analizados, los microorganismos más frecuentes fueron Lactobacillus spp., difteroides, cocobacilos gram variables compatibles con Gardnerella spp., y Mobiluncus spp. Se destaca la baja frecuencia de hongos y Trichomonas spp. y la ausencia de Neisseria spp., en las mujeres estudiadas.

Se halló que $75 \%$ de mujeres tenían Lactobacillus spp. y como se sabe de ellos por la literatura, estos hacen parte de la flora vaginal normal de mujeres en edad fértil $(27,28)$, las cuales correspondían a la mayor proporción de nuestra población. Estos resultados tienen concordancia con un estudio realizado a 302 pacientes de una clínica en Seattle (USA,) donde se halló Lactobacillus spp. en $71 \%(\mathrm{n}=215)$
Tabla 1. Características clínicas de la población.

\begin{tabular}{|c|c|c|c|}
\hline \multicolumn{3}{|l|}{ Criterio } & \multirow{3}{*}{$\begin{array}{c}\begin{array}{c}\text { Frecuencia } \\
\text { relativa }\end{array} \\
16 \% \\
83 \%\end{array}$} \\
\hline \multirow[t]{8}{*}{ Síntomas } & \multirow[t]{2}{*}{ Prurito } & SI & \\
\hline & & NO & \\
\hline & \multirow[t]{2}{*}{ Sinusorragia } & SI & $2 \%$ \\
\hline & & NO & $98 \%$ \\
\hline & \multirow[t]{2}{*}{ Dispareunia } & SI & $28 \%$ \\
\hline & & NO & $71 \%$ \\
\hline & \multirow[t]{2}{*}{ Dolor pélvico } & SI & $8 \%$ \\
\hline & & NO & $91 \%$ \\
\hline \multirow[t]{7}{*}{ Flujo vaginal } & \multirow[t]{2}{*}{ Aspecto } & Homogéneo & $65 \%$ \\
\hline & & Grumoso & $26 \%$ \\
\hline & \multirow[t]{5}{*}{ Color } & Blanco & $73 \%$ \\
\hline & & Amarillo & $11 \%$ \\
\hline & & Transparente & $8 \%$ \\
\hline & & Grisáceo & $4 \%$ \\
\hline & & Verdoso & $0 \%$ \\
\hline
\end{tabular}

Tabla 2. Hallazgos químicos, citológicos y microbiológicos del flujo vaginal.

\begin{tabular}{|l|c|c|}
\hline Criterio & X $\pm \mathbf{D E}$ & Me(RIQ) \\
\hline $\mathrm{pH}$ & $5,1 \pm 0,8$ & $4,9(4,6-5,8)$ \\
\hline & Frecuencia absoluta & Frecuencia relativa \\
\hline Test aminas positivo & 81 & $16 \%$ \\
Células guía & 105 & $21 \%$ \\
Reacción leucocitaria & 468 & $92 \%$ \\
Trichomonas spp. & 4 & $0,80 \%$ \\
Lactobacillus spp. & 382 & $75 \%$ \\
Corineformes & 301 & $59 \%$ \\
Blastoconidias & 27 & $5 \%$ \\
Pseudomicelios & 30 & $6 \%$ \\
Cocobacilos gramvariables & 111 & $22 \%$ \\
Mobiluncus spp. & 71 & $14 \%$ \\
\hline X: Media. DE: Desviación Estándar. ME: Mediana. RIQ: Rango intercuartil. \\
\hline \multicolumn{2}{|c|}{} \\
\hline
\end{tabular}

(29). Estas llamativas frecuencias de Lactobacillus spp. encontradas tanto en población general como en pacientes en ambos estudios pueden explicarse por casos en los cuales pacientes consultantes tienen al mismo tiempo Lactobacillus spp., y otros microorganismos patógenos.

En nuestras participantes, la prevalencia de Corynebacterium spp. fue 59\% y se asoció con reacción leucocitaria, donde se observó una mayor frecuencia de la presencia de este microorganismo con esta reacción en cantidad escasa (49\%) o moderada (40\%), lo que de acuerdo con las recomendaciones de la ASM no se interpretarían como proceso patológico (25). En artículos de otros autores, reportan presencia de Corynebacterium spp. hallado en el tracto genital femenino de mujeres embarazadas produ- 
Tabla 3. Análisis bivariado de bacterias corineformes y características físicas y microbiológicas del flujo vaginal.

\begin{tabular}{|c|c|c|c|c|c|c|}
\hline & & \multicolumn{2}{|c|}{ Negativo } & \multicolumn{2}{|c|}{ Positivo } & \multirow[t]{2}{*}{ Vp } \\
\hline & & $\#$ & $\%$ & $\#$ & $\%$ & \\
\hline \multirow[t]{5}{*}{ Color del flujo $(\mathrm{N}=498)$} & Blanco & 152 & 41 & 216 & 59 & $0,044^{\ddagger}$ \\
\hline & Amarillo & 21 & 37 & 36 & 63 & \\
\hline & Transparente & 20 & 51 & 19 & 49 & \\
\hline & Grisáceo & 10 & 56 & 8 & 44 & \\
\hline & Verdoso & 0 & 0 & 2 & 100 & \\
\hline \multirow[t]{2}{*}{ Test aminas $(\mathrm{N}=509)$} & Positivo & 150 & 35 & 278 & 65 & $0,000^{* * *}$ \\
\hline & Negativo & 59 & 73 & 22 & 27 & \\
\hline \multirow[t]{2}{*}{ Células guía (N=510) } & Presentes & 83 & 79 & 22 & 21 & $0,000^{p+*}$ \\
\hline & Ausentes & 126 & 31 & 279 & 69 & \\
\hline \multirow[t]{2}{*}{ Lactobacillus spp. $(\mathrm{N}=510)$} & Presentes & 120 & 32 & 261 & 68 & $0,000^{\dagger *}$ \\
\hline & Ausentes & 89 & 69 & 4031 & & \\
\hline \multirow[t]{2}{*}{ Hongos $(\mathrm{N}=510)$} & Presentes & 9 & 25 & 27 & 75 & $0,043^{+}$ \\
\hline & Ausentes & 200 & 42 & 274 & 58 & \\
\hline \multirow[t]{2}{*}{ Cocos gramvariables $(\mathrm{N}=510)$} & Presentes & 89 & 80 & 22 & 20 & $0,000^{* * *}$ \\
\hline & Ausentes & 120 & 30 & 279 & 70 & \\
\hline \multirow[t]{2}{*}{ Mobiluncus spp. $(\mathrm{N}=510)$} & Presentes & 57 & 80 & 14 & 8 & $0,000^{* * *}$ \\
\hline & Ausentes & 152 & 35 & 287 & 65 & \\
\hline \multirow[t]{2}{*}{ Reacción leucocitaria $(\mathrm{N}=510)$} & Presentes & 181 & 39 & 286 & 61 & $0,001^{* * *}$ \\
\hline & Ausentes & 28 & 65 & 15 & 35 & \\
\hline \multirow[t]{2}{*}{ Vaginosis bacteriana $(\mathrm{N}=281)$} & Presente & 30 & 79 & 8 & 21 & $0.000^{k * *}$ \\
\hline & Ausente & 96 & 40 & 147 & 60 & \\
\hline \multirow{2}{*}{\multicolumn{2}{|c|}{$\mathrm{pH}(\mathrm{N}=281)$}} & $\mathrm{X} \pm \mathrm{DE}$ & Me & $\mathrm{X} \pm \mathrm{DE}$ & Me & \\
\hline & & $5,3 \pm 0,8$ & $5,5(4,6-6,1)$ & $5,0 \pm 0,7$ & $4,6(4,5-5,5)$ & $0,000^{\mathrm{t}}$ \\
\hline
\end{tabular}

ciendo complicaciones en la gestación, incluyendo aborto espontáneo, parto prematuro, y bajo volumen de líquido amniótico, sin encontrarse ningún otro microorganismo patógeno significativo (30 - 32); estos casos sugieren que Corynebacterium spp. podría estar comportándose como un patógeno oportunista en el embarazo, contrario a lo encontrado en la mayor proporción de participantes de nuestro estudio; sin embargo, es importante analizar detalladamente que del total de nuestra población, $6 \%$ tenía Corynebacterium spp. con reacción leucocitaria abundante y de estas mujeres, en $67 \%$ no había presencia de otro microorganismo patógeno (12 mujeres de 18 ).

Por otro lado, con el fin de explorar alguna asociación de bacterias corineformes (difteroides) con síntomas ginecológicos tales como dispareunia, dolor pélvico, sinusorragia y prurito $(33,34)$, no se encontró asociación alguna con estas variables. De acuerdo con nuestra búsqueda bibliográfica, esta es la primera vez que se hace un estudio donde se explora la asociación de las bacterias corineformes con la sintomatología de las mujeres que las presentan.

La frecuencia hallada de cocobacilos gram variables compatibles con Gardnerella spp., fue $22 \%$, un porcentaje llamativo, tratándose de mujeres de población general; en otro estudio nuestro, encontramos una prevalencia de Gardnerella spp. de 39\% en pacientes (35). Diferentes autores han observado que este microorganismo tiene incidencia alta entre mujeres sexualmente activas (36 - 38).

Por otro lado, la vaginosis bacteriana (VB) caracterizada por un flujo homogéneo, presencia de células guía, test de aminas positivo y un $\mathrm{pH}$ por encima de $4.5(39,40)$, presentó una frecuencia de $7.4 \%$ en nuestro estudio. La VB ha estado fuertemente asociada con complicaciones del embarazo, partos prematuros, bajo peso al nacer y algunas complicaciones ginecológicas (41). En un estudio de 136 mujeres que fueron a consulta ginecológica se halló VB en $25 \%(n=34)$ de ellas (33).

Otros investigadores han demostrado que algunas prácticas sexuales son factores predisponentes para adquirir una infección de transmisión sexual y el intercambio de flora normal (42), por esta razón analizamos si algunos hábitos del comportamiento sexual tales como la práctica de sexo oral y anal, y tener relaciones sexuales sin condón, estarían asociados a la alta frecuencia de Corynebacterium spp. A pesar de haber encontrado que a las mujeres analizadas les practicaban sexo oral, anal y tenían relaciones genitales sin condón en el mes anterior a la toma de la muestra (61, 
10 y $58 \%$ respectivamente). Estos factores no tuvieron asociación con la presencia de Corynebacterium spp. en nuestros hallazgos.

El 9\% de las mujeres usaron duchas vaginales pero esta práctica no estuvo relacionada con la presencia de bacterias corineformes; en otros artículos este hábito podría ser un factor predisponente para modificar la flora microbiana normal e incrementar la incidencia de las infecciones (43).

Los hallazgos de este estudio deben interpretarse con cautela dado que no fue una investigación de nivel analítico que pueda establecer causalidad y la muestra no es representativa del universo abordado.

\section{Conclusiones}

Se quiso con este estudio determinar la prevalencia de Corynebacterium spp. y explorar alguna relación entre éste, la clínica y los hábitos sexuales de la población estudiada.

La prevalencia de Corynebacterium spp. fue $59 \%$ y se encontró asociado a reacción leucocitaria; no se asoció a comportamientos sexuales específicos ni sintomatología ginecológica. En la mayoría de las participantes con esta bacteria se interpretó como acompañante de la flora normal vaginal, sin embargo, en 18 mujeres se encontró sugestiva de causar infección vaginal sola o asociada a otro microorganismo patógeno (12 y 6 mujeres respectivamente).

\section{Declaración de fuentes de financiación}

Este estudio fue financiado por el Comité Nacional de Investigación (CONADI) de la Universidad Cooperativa de Colombia y por el Centro de Investigación (CICMA) de la Institución Universitaria Colegio Mayor de Antioquia.

\section{Declaración de conflictos de interés}

Los autores declaramos no tener conflictos de interés en el estudio realizado. Todos los autores declaramos no haber recibido patrocinio u honorarios por entidades diferentes a las financiadoras del estudio en relación al mismo.

Todos los autores declaramos que no tenemos vínculos laborales o financieros con casas farmacéuticas ni otras empresas que constituyan conflictos de interés para este estudio.

\section{Referencias}

1. Brosnahan AJ, Merriman JA, Salgado PW, Ford B, Schlievert PM. Enterococcus faecalis Inhibits Superantigen Toxic Shock Syndrome Toxin-1-Induced Interleukin-8 from Human Vaginal Epithelial Cells through Tetramic Acids. PLoS One. 2013; 8 (4): e61-255.

2. Sanjay KS, Kathryn AB, Kurt DR. Corynebacterium nigricans sp. nov.: Proposed Name for a Black-Pigmented Corynebacterium Species Recovered from the Human Female Urogenital. J ClinMicrobiol. 2003; 41 (9): 4353-4358.

3. Lamont RF, Sobel JD, Akins RA, Hassan SS, Chaiworapongsa T, Kusanovic JP, et al. The vaginal microbiome: new information about genital tract flora using molecular based techniques. BJOG. 2011; 118 (5): 533-549.

4. Hyman RW, Fukushima M, Diamond L, Kumm J, Giudice L.C, Davis R.W. Microbes on the human vaginal epithelium. PNAS. 2005; 102 (22): 7952-7.

5. Martinez PM, Castro EG, Aguilera AG. Lactobacillus species isolated from vaginal secretions of healthy and bacterial vaginosis-intermediate Mexican women: a prospective study. BMC Infect Dis. 2013; (13): 189.

6. Gamiño AA, Barrios CM, Cárdenas PL, Anaya VF, Padilla VF. Flora Normal, Probióticos y Salud Humana. Acta universitaria. 2005; (15): 5, pp. 34-40.

7. Coyle MB and Lipsky BA. Coryneform bacteria in infectious diseases: clinical and laboratory aspects. ClinMicrobiol Rev. 19903 (3): 227-246.

8. Turovskiy Y, Sutyak NK, Michael L. Chikindas LM. The etiology of bacterial vaginosis. J Appl Microbiol. 2011; 110 (5): 1105-1128.
9. Chávez N, Molina H, Sánchez J, Geyale B, Sánchez SE. Duchas vaginales y otros riesgos de vaginosis bacteriana. Rev Peru Med Exp Salud Pública. 2009; 26 (3): 299-306.

10. Castro J, Henriques A, Machado A,Henriques M, Jefferson KK, Cerca N. Reciprocal Interference between Lactobacillus spp. and Gardnerella vaginalis on Initial Adherence to Epithelial Cells. Int J Med Sci. 2013; 10 (9): 1193-1198.

11. Gutman RE, Peipert JF, Weitzen S, Blume J. Evaluation of clinical methods for diagnosing bacterial vaginosis. Obstet Gynecol. 2005; 105: 551-556.

12. Aminzadeh Z, Fadaeian A. Reactive Arthritis Induced by Bacterial Vaginosis: Prevention with an Effective Treatment. Int J Prev Med. Jul 2013; (7) 4: 841-844.

13. Mastromarino P, Vitali B, Mosca L. Bacterial vaginosis: a review on clinical trials with probiotics. New microbiológica. 2013; 36: 229-238.

14. Caballero PR, Batista MR, Cué BM, Ortega GL, Rodríguez BM. Vaginosis bacteriana. Resumed 2000; 13 (2): 63-75.

15. Venezia J, Cassiday P.K, Marini R.P, Shen Z,Buckley E.M, Peters Y, et al. Characterization of Corynebacterium species in macaques. J Med Microbiol. 2012; 61 (10): 1401-1408.

16. Fernández M. Identificación y poder patógeno de microorganismos del género "corynebacterium" aislados de muestras clínicas; 2009 [acceso Junio 23 del 2013]. Disponible en: http://eprints.ucm.es/11115/

17. David HM. The Microbiota of the Vagina and Its Influence on Women's Health and Disease. Am J Med Sci. 2012; 343 (1): 2-9.

18. Reddy BS, Chaudhury A, Kalawat U, Jayaprada R, Reddy GS, Ramana B.V. Isolation, speciation, and antibiogram of clinically relevant non-diphtherial $\mathrm{Co}$ rynebacteria (Diphtheroids). Indian Journal of Medical Microbiology. 2012; 30: $52-57$.

19. Atieh K, Didier R, Bernard LS. RpoB Gene Sequencing for Identification of Corynebacterium Species. J ClinMicrobiol. 2004; 42 (9): 3925-3931.

20. Martín R, Soberón N, Vázquez F, Evaristo SJ. La microbiota vaginal: composición, papel protector, patología asociada y perspectivas terapéuticas. Enferm Infecc MicrobiolClin. 2008; (26) 3: 160-7.

21. Adnan AA, Charles JC, Robin P. Identification of Non-diphtheriae Corynebacterium by Use of Matrix-Assisted Laser Desorption Ionization-Time of Flight Mass Spectrometry. J ClinMicrobiol. 2012; 50 (1): 160-163.

22.Bernard K. The Genus Corynebacterium and Other Medically Relevant Coryneform-Like Bacteria. J Clin Microbiol. 2012; 50 (10): 3152-3158.

23. Cazanave C, Greenwood K.E, Hanssen A.D, Pate R. Corynebacterium Prosthetic Joint Infection. J ClinMicrobiol. May 2012; 50 (5): 1518-1523.

24. Dalal A, Urban C, Segal MS. Endocarditis due to Corynebacterium amycolatum J Med Microbiol. October 2008; (57): 10,1299-1302.

25. Funke G, Von Graevenitz A, Clarridge III JE, Bernard KA. Clinical microbiology of Coryneform bacteria. Clin Microbiol Rev. 1997; (10) 1: 125-129.

26.Castaño-Orozco ME, Ossa-Giraldo AC, Úsuga-Perilla X, Gil G. Prevalencia de Corynebacterium sp. en mujeres consultantes de una I.P.S de la ciudad de Medellín durante 2006-2012. En: CD-ROM de Memorias: $12^{\circ}$ Congreso Internacional del Colegio Nacional de Bacteriología CNB-Colombia. Bogotá: Colegio Nacional de Bacteriología CNB; 2012

27. Romero R, Hassan SS, Gajer P, Tarca AL, Fadrosh DW, Nikita L, et al. The composition and stability of the vaginal microbiota of normal pregnant women is different from that of non-pregnant women. Microbiome. 2014. 3; (2): 1-4.

28. Vásquez A, Jakobsson T,Ahrné S, Forsum U, Molin G. Vaginal Lactobacillus Flora of Healthy Swedish Women. J Clin Microbiol. 2002; (40) 8: 2746-2749.

29. Antonio MA, Hawes SE, Hillier SL. The Identification of Vaginal Lactobacillus Species and the Demographic and Microbiologic Characteristics of Women Colonized by These Species. J Infect Dis. 1999; (180) 6: 1950-1956.

30. Shukla SK, Bernard KA, Harney M,Frank DN, Reed KD. Corynebacterium nigricans sp. nov.: Proposed Name for a Black-Pigmented Corynebacterium Species Recovered from the Human Female Urogenital Tract. J Clin Microbiol. Sep 2003; (41) 9: 4353-4358.

31.Shukla SK, Vevea DN, Frank DN, Pace NR, Reed KD. Isolation and Characterization of a Black-Pigmented Corynebacterium sp. from a Woman with Spontaneous Abortion. J Clin Microbiol. Mar 2001; (39) 3: 1109-1113.

32.Shukla SK, Harney M, Jhaveri B, Andrews K, Reed KD. Is a Black Pigmented Corynebacterium Species an Opportunistic Pathogen during Pregnancy? Literature Review and Report of 3 New Cases. Clin Infect Dis. 2003; (37) 6: 834-837. 
33. González C, Moreno MA, Nieves B, Flores A, Chille A, Carrero S, Rangel E. Flora vaginal en pacientes que asisten a consulta ginecológica. Rev Soc Vene Microbiol 2006; (26): 19-26.

34. Martin DH. The Microbiota of the Vagina and Its Influence on Women's Health and Disease. Am J Med Sci. 2012; (343)1: 2-9.

35. Ossa-Giraldo AC, Cardona-Arias JA, Úsuga-Perilla X, Gil G. Prevalencia de infecciones vaginales en una I.P.S de Medellín durante 2006-2012. En: CD-ROM de Memorias: $12^{\circ}$ Congreso Internacional del Colegio Nacional de Bacteriología CNB-Colombia. Bogotá: Colegio Nacional de Bacteriología CNB; 2012.

36. Fredricks DN, Fiedler TL, Marrazzo JM. Molecular Identification of Bacteria Associated with Bacterial Vaginosis. N Engl J Med 2005; (353): 1899-1911.

37. Machado A, Jefferson KK, Cerca N. Interactions between Lactobacillus crispatus and Bacterial Vaginosis (BV)-Associated Bacterial Species in Initial Attachment and Biofilm Formation. Int J Mol Sci. Jun 2013; 14 (6): 12004-12012.
38. Ribeiro SR, Ramos M.M, Barbosa CW, Figueiredo TN, Santos OV, Alves FJ, Arraes AR. Clinical and microbiological profile of women with bacterial vaginosis. Rev. Bras. Ginecol. Obstet. 2010; (32): 2.

39. Morris M, Nicoll A, Simms I, Wilson J, Catchpole M. Bacterial vaginosis: a public health review. Intern J Obst \& Gynaeco. May. 2001; (108): 5, 439-450.

40. Gergova R.T, Strateva TV, Mitov IG. Gardnerella vaginalis-associated bacterial vaginosis in Bulgarian women. Braz J Infect Dis, 2013; (17): 313-318.

41. Ibrahim SM, Bukar M, Galadima GB, Audu B.M, Ibrahim HA. Prevalence of bacterial vaginosis in pregnant women in Maiduguri, North-Eastern Nigeria. Niger J Clin Pract. 2014; 17 (2): 154-8.

42. Zhou X, Bent S.J, Schneider MG, Davis CC, Islam MR, Forney L. Characterization of vaginal microbial communities in adult healthy women using cultivation-independent methods. Microbiology. 2004; 150 (8): 2565-73.

43. Wilson J. Managing recurrent bacterial vaginosis. Sex Transm Infect 2004; (80): 8-11. 